Revista de la Escuela de Ciencias de la Educación, año 13, nRo. 12, vol. 2, Julio a diciembre de 2017. Páginas $195-206$. ISSN 1851-6297. ISSN 2362-3349 (EN LINEA). EVALUACIÓN DE COMPETENCIAS UNIVERSITARIAS. ANA MARIA TROTTINI Y MARIA ANGÉLICA FERRANDI.

\title{
EVALUACIÓN DE COMPETENCIAS UNIVERSITARIAS
}

\author{
Ana María Trottini* \\ Universidad Abierta Interamericana \\ anatrottini@hotmail.com \\ María Angélica Ferrandi** \\ Universidad Abierta Interamericana \\ maferrandi@gmail.com
}

Recibido: 30/09/2016 Aceptado: 06/07/2017

\section{Resumen}

El presente artículo expone las conclusiones a las que se arribó en la elaboración de la tesis del Máster de Desarrollo Emprendedor e Innovación (Universidad de Salamanca-España).

Se selecciona una de las categorías, establecidas por la Organización de Cooperación y el Desarrollo Económico, en su Programa para la Evaluación Internacional para Estudiantes (PISA, por sus siglas en inglés).

Partiendo del objetivo de relevar competencias universitarias, se han buscado indicadores sistematizados y reconocidos mundialmente. Para tal fin se ha utilizado además como instrumento, el test MBTI (Myer-Briggs Type Indicator) transferido a un software de Business Intelligence que permite la visualización de cada uno de los aspectos.

Palabras Clave: Competencias universitarias - Habilidades - Inteligencia de Negocios.

* Magister en Política y Gestión de Gobierno de Instituciones de Educación Superior (UNR-UNL-UBA). Especialista en Política y Gestión de Gobierno de Instituciones de Educación Superior (UNR-UNL-UBA). Licenciada en Ciencia Política (UNR). Profesora Adjunta Metodología Investigación Científica. Coordinadora Tesinas- Facultad Ciencias Empresariales- Universidad Abierta Interamericana-Sede Rosario- 2008 y continúa.

** Licenciada en Estadística (UNR). Asesora en Fundación Camino. Miembro ingresante RED-DEES Red de Educación Superior (Cuba) UAI. 
Revista de la Escuela de Ciencias de la Educación, año 13, nRo. 12, vol. 2, Julio a diciembre de 2017. Páginas $195-206$. ISSN 1851-6297. ISSN 2362-3349 (EN LINEA). EVALUACIÓN DE COMPETENCIAS UNIVERSITARIAS. ANA MARIA TROTTINI Y MARIA ANGÉLICA FERRANDI.

\section{Abstract}

This paper presents the conclusions that were reached in the preparation of the thesis of Master of Entrepreneurial Development and Innovation (University of Salamanca-Spain).

One of the categories established by the Organization for Economic Cooperation and Development, in its Programme for International Student Assessment (PISA acronym in English) is selected.

Starting with the aim of relieving college competition, they have sought systemized indicators and recognized worldwide. For this purpose has also been used as a tool, the test MBTI (Myer-Briggs Type Indicator) transferred to Business Intelligence software that allows viewing of each of the aspects.

Key words: University competence - Abilities - Business Intelligence.

\section{Objetivos}

Identificar técnicas socialmente normalizadas, que permitan medir competencias sociales.

Diseñar estrategia de procesamiento de información que permita abordar las competencias, utilizando una herramienta de inteligencia de negocios.

Recopilar datos relevantes y significativos de competencias sociales, que son abordadas en la formación del alumno universitario.

\section{Introducción}

De Vincenzi, A. (2011) expresa claramente, respecto a la formación pedagógica del profesor universitario:

La función primaria de la universidad es la de formar profesionales competentes, cada vez más preparados para dar respuestas a las necesidades de la comunidad en la que viven, dando respuesta a las exigencias del contexto socioprofesional, como así también educar para el desarrollo personal y para el mejoramiento de la calidad de vida. Esto supone que los programas curriculares contemplen una formación humanística sustentada en valores que contribuyan al desarrollo de habilidades personales (autoestima, autoafirmación, asertividad, entre otras) y sociales (solidaridad, cooperación, sensibilidad social, entre otras). Dichas habilidades deben ser contempladas en la definición del perfil del docente universitario, en tanto que modelo educativo para sus alumnos (Vincenzi, 2011, p.7).

En la actualidad uno de los retos principales tanto para profesionales como investigadores es ser puentes para que los futuros profesionales se encuentren sostenidos por habilidades sociales, para lo cual es fundamental atender al proceso de formación de competencias. 
Revista de la Escuela de Ciencias de la Educación, año 13, nRo. 12, vol. 2, Julio a diciembre de 2017. Páginas 195-206. ISSN 1851-6297. ISSN 2362-3349 (EN LINEA). EVALUACIÓN DE COMPETENCIAS UNIVERSITARIAS. ANA MARIA TROTTINI Y MARIA ANGÉLICA FERRANDI.

Para tal fin, se trabaja en la asignatura Seminario de Aplicación Profesional de la carrera Ingeniería en Sistemas de la Facultad de Tecnología Informática, sede Rosario de la Universidad Abierta Interamericana, desde al año 2011.

Durante el año 2014, se ha investigado a partir del concepto de las competencias universitarias que se expresa en el Proyecto Tunning América Latina (2004-2007) y esta investigación se ha enmarcado en el contexto de competencias a partir del Resumen Ejecutivo elaborado en 1997, por los miembros de la Organización para la Cooperación y el Desarrollo Económico (OECD, por sus siglas en inglés y OCDE, en español) al lanzar el Programa para la Evaluación Internacional para Estudiantes (PISA, por sus siglas en inglés).

Así es como la OCDE et al. (2006), en su Resumen Ejecutivo hace referencia a la competencia: "Interactuar en grupos heterogéneos" (OCDE, 2006, p.11).

Según la Real Academia Española, competencia es "acción o tarea que entraña dificultad y cuya ejecución requiere decisión y esfuerzo".

Teniendo en cuenta lo expresado por Ángel Díaz Barriga (2011) que analizó el término de referencia, hoy día, el mismo, tomó alta trascendencia, especialmente en el Nivel de la Educación Superior y está relacionado con el hecho de trabajar en equipo y saber relacionarse, esto incluye la noción de flexibilidad y capacidad de resolver situaciones.

En esta misma línea, en 2002, tomadas desde el enfoque funcional y sistémico, la Red EURYDICE (Red Europea de Información en Educación) incorpora el concepto para la reforma de la Educación Básica, considerando que con ello se busca "preparar a los jóvenes para los desafíos que presenta la sociedad de la información" (EURYDICE, 2002, p.4). En algún sentido pensamos que este concepto, si bien no logra una traducción específica, en perspectiva se tradujo con claridad en la pregunta de la prueba PISA. Un esfuerzo singular por buscar que un individuo no reproduzca la información sino que emplee la misma en la resolución de problemas lo más cercano posible a la realidad.

En esta oportunidad se ha elegido la competencia: "Interactuar en grupos heterogéneos", expresada por PISA, como variable intangible que debe ser evaluada al momento de realizar mejoras curriculares en el ámbito universitario para el desempeño en una sistémica vinculable a las organizaciones sociales.

Se plantea lo expuesto por Sergio Tobón:

Las competencias son un enfoque para la educación en general, las competencias focalizan en unos aspectos específicos de la docencia, del aprendizaje y de la evaluación, como son: 1) la integración de los conocimientos, los procesos cognoscitivos, las destrezas, las habilidades, los valores y las actitudes en el desempeño ante actividades y problemas; 2) la construcción de los programas de formación acorde con los requerimientos disciplinares, investigativos, profesionales, sociales, ambientales y laborales del contex- 
Revista de la Escuela de Ciencias de la Educación, año 13, nRo. 12, vol. 2, Julio a diciembre de 2017. Páginas $195-206$. ISSN 1851-6297. ISSN 2362-3349 (EN LiNEA). EVALUACIÓN DE COMPETENCIAS UNIVERSITARIAS. ANA MARÍA TROTTINI Y MARÍA ANGÉLICA FERRANDI.

to; 3) la orientación de la educación por medio de estándares e indicadores de logros (Tobón, 2006, pp.1-2).

Siguiendo con esta línea de pensamiento, se propone abordar la investigación seleccionando una de las competencias de PISA, establecidas en su Resumen Ejecutivo et. al (2006): "Competencia Categoría 2: Interactuar en grupos heterogéneos; Competencia 2A: La habilidad de relacionarse bien con otros. Competencia 2B: La habilidad de cooperar" (pp. 11-12).

Estas competencias si bien son consideradas por PISA como objetivo para monitorear a los alumnos al final de la escolaridad obligatoria, se considera que son altamente pertinentes para realizar su análisis en el ámbito universitario. La OCDE (2006), expresó que "las competencias no pueden abordarse a partir de decisiones arbitrarias sobre las cualidades personales y las destrezas cognitivas deseables; son una consideración cuidadosa de los prerrequisitos psicosociales para un buen funcionamiento de la sociedad" (p.5).

Por tal motivo se ha seleccionado un instrumento como es la teoría de Jung que se ha vuelto muy popular desde la década de los 80 como instrumento psicológico muy utilizado, diseñado para ayudar a las personas a identificar algunas de sus preferencias personales más importantes.

Myers \& Briggs (1976), diseñó el instrumento MTBI (Myer-Briggs Type Indicator) para establecer preferencias individuales e identificar las diferencias entre las personas principalmente en aspectos de la personalidad, se basó en la teoría tipológica de Jung y consta de 72 preguntas dicotómicas.

En forma análoga, se propone procesar la información obtenida de las preferencias individuales del alumno, tomadas como "subjetividades" personales, que permitirán identificar los rasgos de la competencia, que el egresado cuenta para lograr su inserción en las organizaciones sociales.

Dichos aspectos se toman y vuelcan a un software de inteligencia de negocios, Business Intelligence, a fin de brindar dichas características para generar espacios reflexivos y relevantes para la toma de decisiones.

El Ministerio de Educación, Cultura y Deporte de España (2003) manifiesta en aquellos años, como nuevos retos del sistema universitario español:

Una adecuada comprensión de los cambios que han de introducirse en la estructura y en el funcionamiento de las enseñanzas universitarias sólo resulta posible concibiéndolos como eficaces instrumentos orientados al logro de una mejora de la calidad y de una plena adecuación de estas enseñanzas a las exigencias que una sociedad del conocimiento demanda (p.2).

"El objetivo básico del software Business Intelligence es apoyar de forma sostenible y continuada a las organizaciones para mejorar su competitividad, facilitando la información necesaria para la toma de decisiones". Cano, Josep Lluís (2007).

La OCDE expresa (2006) que: 
Revista de la Escuela de Ciencias de la Educación, año 13, nRo. 12, vol. 2, Julio a diciembre de 2017. Páginas $195-206$. ISSN 1851-6297. ISSN 2362-3349 (EN LINEA). EVALUACIÓN DE COMPETENCIAS UNIVERSITARIAS. ANA MARIA TROTTINI Y MARIA AngÉLICA FERRANDI.

Las personas necesitan respaldarse en competencias permitiendo involucrarlos en un mundo en constante cambio, complejo e independiente. Estas competencias deben ser aptas en un mundo donde la tecnología cambia rápido y de forma continua requiriendo capacidad de adaptación. Las relaciones interpersonales requieren mayor contacto con personas diferentes a uno. Lo mencionado anteriormente marca desafíos individuales y globales (p.6).

En la actualidad los profesionales requieren contar con competencias profesionales, técnicas y de gestión que incluyan habilidades de adaptación, interpretación del contexto socio-cultural donde llevan a cabo su ejercicio profesional.

Las competencias sociales son las que acompañarán al alumno en su desempeño profesional.

La pregunta que orienta al estudio: ¿Es posible medir competencias universitarias requeridas para el desempeño profesional del egresado, utilizando una herramienta de Inteligencia de Negocios?

\section{Metodología}

Participantes: Alumnos 5to. Año de la carrera Ingeniería en Sistemas 2015.

Asignatura seleccionada: Seminario de Aplicación Profesional.

Edad promedio: 29 años.

Perfil socioeconómico medio-alto ya que se trata de una Universidad Privada. Alumnos que trabajan: $53,85 \%$.

Técnicas de recolección de datos: Test Jung-Myers Briggs (MTBI).

Cantidad de respuestas: 47 (cuarenta y siete).

Fecha: Primer cuatrimestre del año 2015.

Procedimiento:

Se solicita a los alumnos que respondan las preguntas del indicador Test Jung-Myers Briggs (MTBI) que están relacionadas directamente con la manera preferida de usar su percepción y juicio, mediante dos respuestas: sí o no.

Teniendo en cuenta la competencia Categoría 2: Interactuar en grupos heterogéneos de PISA, se identifican aquellas preguntas dicotómicas de MTBI que permiten aproximarse para el desarrollo de habilidades.

Se selecciona la Competencia Categoría 2: Interactuar en grupos heterogéneos, incluyendo las dos competencias de PISA: La habilidad de relacionarse bien con otros y la habilidad de cooperar, en esta primera instancia.

Teniendo en cuenta ambas habilidades se han seleccionado, de las 72 preguntas dicotómicas de MTBI, sólo aquellas que retroalimenten a estas dos habilidades, realizando vinculaciones directas entre cada una de ellas con sus habilidades intrínsecas y las preguntas del test MTBI. Se han respetado las numeraciones propias del test para que sea posible identificar claramente la relación entre estas dos técnicas socialmente reconocidas, así como también 
se han establecido números a las subcategorías para posibilitar su lectura, interpretación y luego reflexión. Las mismas son las siguientes:

Competencia 2A: La habilidad de relacionarse bien con otros.

2-A.1: Empatía.

36. Empatizar fácilmente con las preocupaciones de otras personas.

2-A.2: Manejo efectivo de las emociones.

19. Tus acciones son frecuentemente influenciadas por tus emociones.

27. Expresas frecuente y fácilmente tus sentimientos y emociones.

64. Eres fácilmente afectable por emociones fuertes.

Competencia 2B: La habilidad de cooperar.

2-B.1: Habilidad de presentar y escuchar ideas de otros.

69. Te resulta fácil comunicarte en situaciones sociales.

2-B.2: Entendimiento de la dinámica del debate y el seguimiento de una agenda.

42. Los plazos te parecen de relativa y NO de absoluta importancia.

47. Obtienes placer al poner las cosas en orden.

2-B.3: La Habilidad de construir alianzas tácticas y sostenibles.

54. Intentas permanecer firme a tus principios.

2-B.4: La Habilidad de negociar.

22. Ayudas a la gente sin pedir nada a cambio.

2-B.5: La capacidad de tomar decisiones que permitan diferentes opiniones.

14. Prefieres actuar inmediatamente en lugar de especular sobre varias opciones.

Se confecciona una planilla de doble entrada: preguntas del indicador/ respuesta del alumno.

Se importan los datos en software de Business Intelligence.

Se determinan distintas gráficas para mostrar las dimensiones de la competencia

Categoría 2: Interactuar en grupos heterogéneos, discriminada en la competencia 2A: La habilidad de relacionarse bien con otros y 2B: La habilidad de cooperar, correspondiente a PISA. 
Revista de la Escuela de Ciencias de la Educación, año 13, nRo. 12, vol. 2, julio a diciembre de 2017. Páginas $195-206$. ISSN 1851-6297. ISSN 2362-3349 (EN LINEA). EVALUACIÓN DE COMPETENCIAS UNIVERSITARIAS. ANA MARÍA TROTTINI Y MARIA ANGÉLICA FERRANDI.

\section{Resultados}

En el contexto sistémico de la competencia: "Interactuar en grupos heterogéneos", se han obtenido dos gráficas representativas de cada una de las habilidades expresadas por PISA.

En la Gráfica I, se observa que en la Competencia: "La habilidad de relacionarse bien con otros", sus respectivas subcategorías enumeradas y su vinculación con los indicadores del test MTBI seleccionados previamente.

Se han obtenido los siguientes porcentajes de participación:

Competencia Categoría 2: Interactuar con grupos heterogéneos.

2-A: La habilidad de relacionarse con otros.

2-B: La habilidad de cooperar.

2-C: La habilidad de manejar y resolver conflictos.

\section{Listado de preguntas con cantidad de respuesta según criterio.}

$\begin{array}{ll}\text { Preguntas } & \text { SI NO }\end{array}$

\begin{tabular}{|l|l|l|}
\hline 19. Tus acciones son frecuentemente influenciadas por tus emociones & 30 & 17 \\
\hline 27. Expresas frecuente y fácilmente tus sentimientos y emociones & 19 & 28 \\
\hline 36. Empatizas fácilmente con las preocupaciones de otras personas & 35 & 12 \\
\hline 64. Eres fácilmente afectable por emociones fuertes & 32 & 15 \\
\hline
\end{tabular}
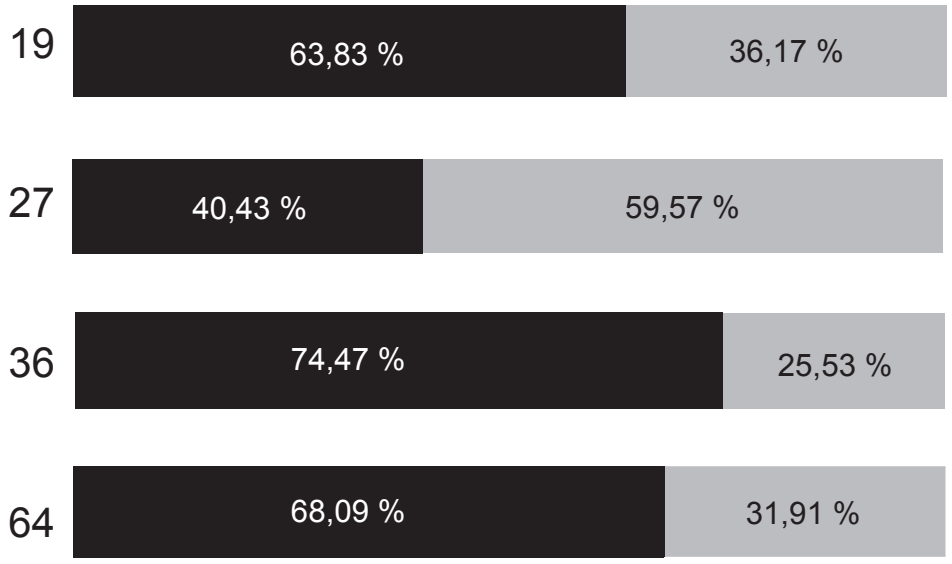

PORC SI

PORC NO

Gráfica I 
Revista de la Escuela de Ciencias de la Educación, año 13, nRo. 12, vol. 2, julio a diciembre de 2017. Páginas $195-206$. ISSN 1851-6297. ISSN 2362-3349 (EN LINEA).EVALUACIÓN DE COMPETENCIAS UNIVERSITARIAS. ANA MARIA TROTTINI Y MARÍA ANGÉLICA FERRANDI.

\section{2-A.1: Empatía.}

36. Empatizas fácilmente con las preocupaciones de otras personas $74,47 \%$. 2-A.2: Manejo efectivo de las emociones.

19. Tus acciones son frecuentemente influenciadas por tus emociones $63,83 \%$.

27. Expresas frecuente y fácilmente tus sentimientos y emociones $40,43 \%$.

64. Eres fácilmente afectable por emociones fuertes 68,09\%.

La segunda gráfica, Gráfica II, hace referencia a la Competencia: "La habilidad de cooperar".

En la misma se pueden observar los siguiente porcentajes de participación expresadas por los alumnos teniendo en cuenta las habilidades expresadas por PISA y su vinculación con los indicadores del test MTBI.

\section{Competencia Categoría 2: Interactuar con grupos heterogéneos.}

2-A: La habilidad de relacionarse con otros.

2-B: La habilidad de cooperar.

2-C: La habilidad de manejar y resolver conflictos.

\section{Listado de preguntas con cantidad de respuesta según criterio.}

Preguntas
\begin{tabular}{|l|l|l|}
\hline $\begin{array}{l}\text { 14. Prefieres actuar inmediatamente en lugar de especular sobre } \\
\text { varias opciones }\end{array}$ & 13 & 34 \\
\hline 42. Los plazos te parecen de relativa y NO de absuluta importancia & 12 & 35 \\
\hline 47. Obtienes placer al poner las cosas en orden & 40 & 7 \\
\hline 54. Intentas permanecer firme a tus principios & 42 & 5 \\
\hline 69. Te resulta fácil comunicarte en situaciones sociales & 34 & 13 \\
\hline
\end{tabular}

PORC SI

PORC NO

69

$63,83 \%$

$36,17 \%$

Gráfica II 
Revista de la Escuela de Ciencias de la Educación, año 13, nRo. 12, vol. 2, julio a diciembre de 2017. Páginas 195-206. ISSN 1851-6297. ISSN 2362-3349 (EN LINEA). EVALUACIÓN DE COMPETENCIAS UNIVERSITARIAS. ANA MARIA TROTTINI Y MARIA ANGÉLICA FERRANDI.

2-B.1: Habilidad de presentar y escuchar ideas de otros.

69. Te resulta fácil comunicarte en situaciones sociales $63,83 \%$.

2-B.2: Entendimiento de la dinámica del debate y el seguimiento de una agenda.

42. Los plazos te parecen de relativa y NO de absoluta importancia $25,53 \%$.

47. Obtienes placer al poner las cosas en orden $85,11 \%$.

2-B.3: La Habilidad de construir alianzas tácticas y sostenibles.

54. Intentas permanecer firme a tus principios $89,36 \%$.

2-B.4: La Habilidad de negociar.

22. Ayudas a la gente sin pedir nada a cambio $97,87 \%$.

2-B.5: La capacidad de tomar decisiones que permitan diferentes opiniones. 14. Prefieres actuar inmediatamente en lugar de especular sobre varias opciones $27,66 \%$.

\section{Conclusiones}

Para la UNESCO, las competencias emprendedoras, entre las que se encuentran las sociales, implican innovación, creación, comunicación y la crítica que son necesarias para actuar y enfrentar como ciudadano responsable la realidad del siglo XXI.

Teniendo en cuenta los objetivos planteados para la actual investigación, tales como la posibilidad de relevar competencias universitarias propuestas, en particular las de formación personalizada de los alumnos, se denota a través de los resultados, un conjunto de las características del perfil del alumnado, que se manifestará en su desempeño profesional.

La importancia de poder realizar aproximaciones, a través de la utilización del software de Business Intelligence, con la selección de algunos indicadores MTBI, entorno a la Competencia: "Interactuar en grupos heterogéneos", de PISA, permite realizar aportes al estado de situación en la formación de competencias sociales universitarias abarcando esencialmente aquellos aspectos que han de estar presentes al enfrentarse a las presiones y demandas diarias, tanto las intrapersonales como habilidades de comprender y de relacionarse con otros, así como también las interpersonales respecto a la habilidad para manejar emociones.

Se observa que en la Competencia: "La habilidad de relacionarse bien con otros" el 74,47\% empatiza fácilmente con las preocupaciones de otras personas, además el $68,09 \%$ es fácilmente afectable por emociones fuertes y que en un $63,83 \%$ sus acciones son frecuentemente influenciadas por sus emociones. Estos porcentajes permiten evidenciar la ponderación de las 
Revista de la Escuela de Ciencias de la Educación, año 13, nRo. 12, vol. 2, JUlio a diciembre de 2017. PÁginas $195-206$. ISSN 1851-6297. ISSN 2362-3349 (EN LINEA). EVALUACIÓN DE COMPETENCIAS UNIVERSITARIAS. ANA MARIA TROTTINI Y MARIA ANGÉLICA FERRANDI.

subjetividades expresadas a la hora de vincularse con el "otro».

El 40,43\% es capaz de expresar frecuente y fácilmente sus sentimientos y emociones. Es menor el porcentaje de expresión de esas emociones que tanto influyen en el momento de vincularnos con el otro sujeto subjetivo.

Brizeida Hernández Sánchez (2015) expresa claramente la importancia de la formación de competencias Sociales: "Como estructuras complejas, ya que se relacionan con todo el funcionamiento psicológico de la persona, guardan relación con el desarrollo de habilidades desde las dimensiones humanas." (p.29)

En la Competencia: "La habilidad de cooperar", el 97,87\% del alumnado ayuda a la gente sin pedir nada a cambio, el $89,36 \%$ intenta permanecer firme a sus principios, lo que le permite evidenciar su habilidad de construir alianzas tácticas y sostenibles.

Por otra parte, el 85,11\%, siente placer al poner las cosas en orden, lo habilita en el seguimiento de una agenda.

En la formación y capacitación en emprendimiento, haciendo referencia a las competencias emprendedoras, Hernández Sánchez (2015) expresa que:

La efectividad constituye un nuevo paradigma del desarrollo que establece un difícil desafío personal y organizacional, se busca incorporar a la personalidad y cada una de las dimensiones de la personalidad. La efectividad personal, basada en la confianza, constituye la relación conmigo mismo, la efectividad interpersonal que son las relaciones que se tienen con los demás.

Las habilidades sociales pueden ser aprovechadas por la mayoría de las personas que desean alcanzar una vida plena (p.49).

Al $63,83 \%$, le resulta fácil comunicarse en situaciones sociales, aunque prefiere actuar inmediatamente en lugar de especular sobre varias opciones, un $27,66 \%$. Claramente pone en evidencia la sociedad de la inmediatez y el menor desarrollo en la habilidad de tomarse el tiempo necesario para escuchar diferentes opiniones a la hora de tomar decisiones.

Además el $25,53 \%$, considera que los plazos tienen relativa y no absoluta importancia.

José Carlos Sánchez García (2015), en la formación y capacitación en emprendimiento, haciendo referencia a la figura del emprendedor, sensibilización y fomento, manifiesta:

Formarse y entrenarse como emprendedor eficaz es un proceso largo y complejo que requiere de un método y de la práctica de ciertos instrumentos.

En primer lugar, se requiere desarrollar habilidades cognitivas, emocionales y sociales en los distintos niveles de educación y capacitación que tenga acceso y hacerlo en forma sistemática durante su desarrollo en la vida familiar y en su paso por el sistema de educación (Sánchez García, 2015, 
Revista de la Escuela de Ciencias de la Educación, año 13, nRo. 12, vol. 2, julio a diciembre de 2017. Páginas 195-206. ISSN 1851-6297. ISSN 2362-3349 (EN LINEA). EVALUACIÓN DE COMPETENCIAS UNIVERSITARIAS. ANA MARIA TROTTINI Y MARIA ANGÉLICA FERRANDI.

\section{p.11).}

Haciendo referencia a la función social de la Universidad, De Vincenzi, A. (2011) considera fundamental considerar el documento titulado "Declaración Mundial sobre la educación superior en el siglo XXI: visión y acción" (1998) y en el comunicado emitido en julio de 2009 como resultado de la Conferencia Mundial sobre la Educación Superior celebrada en París, los que señalan que la universidad tiene por funciones: formar profesionales competentes y comprometidos con los problemas sociales y culturales; investigar y producir conocimientos que contribuyan a dar respuestas interdisciplinarias a los problemas complejos y polifacéticos que afectan a la sociedad; aprovechar el potencial de las TIC tanto para mejorar el proceso educativo como para generar una mayor difusión y acceso al estado actual del conocimiento; desarrollar programas de capacitación del personal docente para mejorar sus competencias didácticas en aras a formar profesionales competentes, críticos y reflexivos (p.6).

En este sentido se propone la continuidad de este relevamiento como puente para generar espacios de reflexión, que permitan diagnosticar el estado de situación, en la formación de competencias sociales, en los futuros profesionales, de manera tal que se puedan realizar los ajustes necesarios anticipadamente y allí abordar una dinámica continua de mejora en los procesos de formación.

Ahora bien, para que ello suceda el sujeto ante todo es Sujeto del Deseo, si no tiene conciencia de ello, no podrá ni siquiera proponerse a sí mismo el desarrollo de las habilidades que requiere para alcanzar sus sueños y que a la vez beneficie el cambio social que requiere esta humanidad.

\section{Referencias bibliográficas}

- Barkley, E. (2007). Técnicas de aprendizaje colaborativo: manual para el profesorado universitario. Madrid: Morata.

- Cano, J. L. (2007). Business intelligence: competir con información. Recuperado de: http://www.dataprix.com.

- De Vincenzi, A. (2011). La formación pedagógica del profesor universitario. Revista Aula. Recuperado de: https://www.uai.edu.ar:443/facultades/desarrollo-e-investigacioneducativos/especializacion-docenciauniversitaria/y60905/La formación pedagógica del profesor universitario. Revista Aula.pdf

- Díaz Barriga, Á. (2011). Competencias en educación. Corrientes de pensamiento e implicaciones para el currículo y el trabajo en el aula. Revista Iberoamericana de Educación Superior. Recuperado de: https://ries.universia.net/article/download/61/120

- Hernández Sánchez, B. (2015). Cátedra de Emprendedores. Módulo: Formación y Capacitación en emprendimiento. Competencias Emprendedoras. España, Salamanca.

- Myers Bryggs, I. (1976). Myers-Briggs Type Indicator (MBTI). Recuperado de: http:// 
www.discoveryourpersonality.com

- OCDE (2006). DeSeCo. La definición y selección de competencias clave. Recuperado de: http://www.deseco.admin.ch

- Real Academia Española. (2012). Diccionario de la lengua española (22.a ed.). Consultado en http://www.rae.es

- Sánchez García, J. C. (2015). Módulo: Formación y Capacitación en emprendimiento. La figura del emprendedor: sensibilización y fomento.

- The Myers \& Briggs Foundation. MBTI Basics. Disponible en la web: http://www. myersbriggs.org

- Tobón, S. (2006). Aspectos básicos de la formación basada en competencias. Recuperado de: http://www.urosario.edu.co/CGTIC/Documentos/aspectos_basicos_ formacion_basada_competencias.pdf 